\title{
Comparison of cloud models for Brown Dwarfs
}

\section{Ch. Helling ${ }^{1}$, A. Ackerman ${ }^{2}$, F. Allard ${ }^{3}$, M. Dehn ${ }^{4}$, P. Hauschildt ${ }^{4}$,

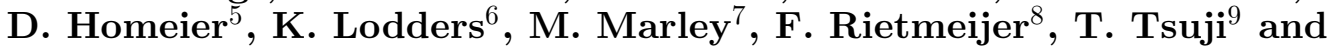 P. Woitke ${ }^{10}$}

${ }^{1}$ SUPA, School of Physics \& Astronomy, University of St Andrews, North Haugh, St Andrews, KY16 9SS, Scotland, UK

email: Christiane.Helling@st-andrews.ac.uk

${ }^{2}$ NASA Goddard Institute of Space Studies, New York, USA

${ }^{3}$ Centre de Recherche Astrophysique de Lyon, Université de Lyon, France

${ }^{4}$ Hamburger Sternwarte, Hamburg, Germany

${ }^{5}$ Universität Göttingen, Institut für Astrophysik, Göttingen, Germany

${ }^{6}$ Planetary Chemsitry Laboratory, Department of Earth and Planetary Sciences,

Washington University, St. Louis, USA

${ }^{7}$ NASA Ames Research Center, Moffett Field, USA

${ }^{8}$ Department of Earth and Planetary Sciences, University New Mexico, Albuquerque, USA

${ }^{9}$ Institute of Astronomy, The University of Tokyo, Mitaka, Tokyo, Japan

${ }^{10}$ UK Astronomy Technology Centre, Royal Observatory, Edinburgh, UK

\begin{abstract}
A test case comparison is presented for different dust cloud model approaches applied in brown dwarfs and giant gas planets. We aim to achieve more transparency in evaluating the uncertainty inherent to theoretical modelling. We show in how far model results for characteristic dust quantities vary due to different assumptions. We also demonstrate differences in the spectral energy distributions resulting from our individual cloud modelling in 1D substellar atmosphere simulations.
\end{abstract}

Keywords. astrochemistry, methods: numerical, stars: atmospheres, stars: low-mass, brown dwarfs

\section{Introduction}

Spectra are windows through which we access the physics and the chemistry of substellar atmospheres down into the planetary regime. Consequently, model atmospheres are needed to produce synthetic spectra. The complexity of such simulations requires the use of approximations and/or analytic simplifications to make them numerically feasible. The input data needed can be frequently incomplete or of limited accuracy, translating into uncertainties in the model results. The formation of clouds in their atmospheres is one of the most fundamental challenges in studies of planets and brown dwarfs. Element depletion and gravitational settling are only two feedback mechanisms arising. We therefore have set out to compare our approaches of cloud formation in substellar atmosphere simulations to achieve more transparency in evaluating the uncertainty inherent to theoretical modelling. We show in how far model results for characteristic dust quantities vary due to different assumptions. We also demonstrate differences in the spectral energy distributions resulting from our individual cloud modelling in 1D substellar atmosphere simulations. 


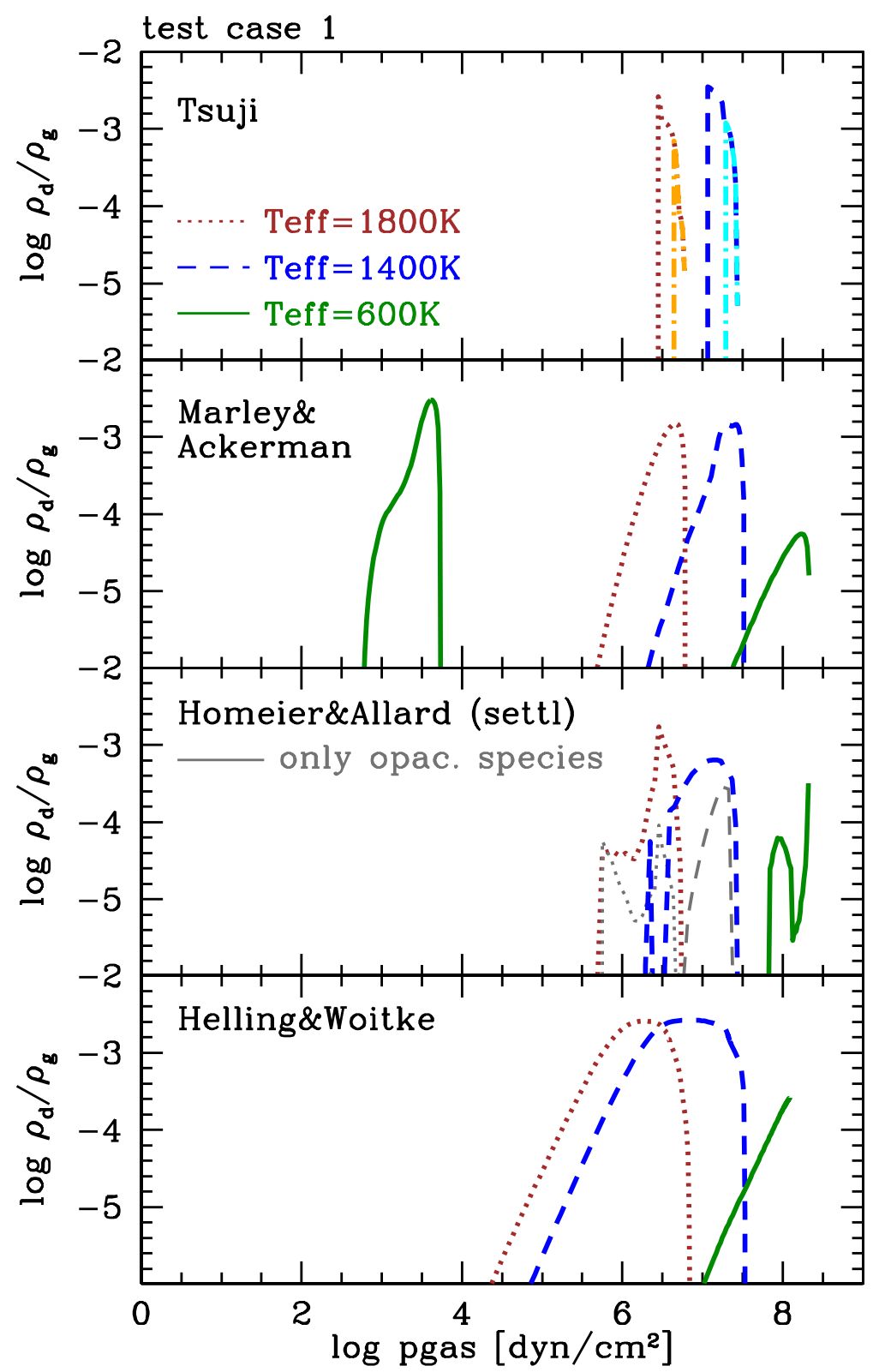

Figure 1. Results for Test case 1 (L and T-dwarf regime): The dust content (dust-to-gas ratio $\left.\rho_{\mathrm{d}} / \rho_{\mathrm{g}}\right)$ of the clouds calculated by different model approaches. Note that in the Tsuji-case two models are plotted for each $\mathrm{T}_{\text {eff }}, \mathrm{T}_{\text {crit }}=1700 \mathrm{~K}$ (red/blue) and $\mathrm{T}_{\text {crit }}=1900 \mathrm{~K}$ (orange $/$ cyan).

\section{Cloud models \& Test cases}

Theoretical models of substellar atmosphere aim to describe the existence of clouds made of small particles and their influence on the atmosphere's energy budget. Basically two model philosophies are followed: The cloud particles hoover inside the atmosphere (Tsuji 2005) or, the particles gravitationally settle, hence disappear from the atmosphere but need to re-form (Ackerman \& Marley 2001; Sudarsky et al. 2003, Woitke \& Helling 2004, Allard et al. 2007). All models aim to produce as accurate as possible synthetic 
spectra by modelling cloud characteristics like the number of dust particles, the material composition of the cloud grains/droplets, material composition, and the size of the individual cloud particles. In general, formation of clouds by condensation would be controlled by kinetic factors, for example induced by turbulence, and amorphous solids would form. Given sufficient time and energy, these amorphous solids will crystallise and form equilibrium solids known as minerals. Phase-equilibrium is adopted in the approaches of Ackerman \& Marley (2001), Tsuji (2005), Allard et al. (2003, 2007), and the kinetic approach is taken by Woitke \& Helling (2004) and Helling, Woitke \& Thi (2008). The dust cloud models involved in the test calculations are summarised by the following simplified description:

Fixed grain size

- grain size $a=$ const

- cloud layer between $\mathrm{T}_{\text {cond }}$ and $\mathrm{T}_{\text {crit }}$

- parameter: $\mathrm{T}_{\text {crit }}$

\section{Time scale comparison}

(Tsuji 2005;

also Burrows et al.)

$-\tau_{\text {growth }}>\tau_{\text {mix }} \Rightarrow$ grain size obtained from $\tau_{\text {growth }}=\tau_{\text {mix }}$

$-\tau_{\text {growth }}<\tau_{\text {mix }} \Rightarrow$ number density of grains obtained from $\tau_{\text {growth }}=\tau_{\text {mix }}$

- convective advection transported into radiative layers according to Ludwig et al. (2002)

\section{Diffusive transport}

- static solution of diffusion equation Marley et al. 2007)

- constant mixing efficiency, homogeneous cloud particles

- parameter: settling efficiency $f_{\text {sed }}$

\section{Top - Down approach}

- quasi-static solution of stationary

(Woitke \& Helling 2003, Helling \& Woitke 2006 ,

moment methods for nucleation, dirty growth/evaporation and element conservation including gravtiational settling

- convective over-shooting parameterized according to Ludwig et al. (2002)

We designed test cases to study difference arising from our different theoretical approach to model clouds in substellar atmosphere simulations. We disentangle our individual cloud models from the complete atmosphere problem (Test case 1) in order to exclude possible feedback amplifications in the entire model atmosphere codes. Only as a second step, we investigate possible differences in the spectral appearance of a given substellar object (Test case 2). The test cases are designed as follows and more details can be found under http://phoenix.hs.uni-hamburg.de/BrownDwarfsToPlanets1/:

\section{Test case 1:}

- compare results from cloud models only

- local quantities given : $\left(\mathrm{T}, \mathrm{p}, v_{\text {conv }}\right)$ structure

- different dust cloud treatment

\section{Test case 2:}

- compare results from complete 1D atmosphere simulation

- global quantities given: $\mathrm{T}_{\text {eff }}=1800 \mathrm{~K}, \log g=5$.

- different model atmosphere codes including different dust cloud treatment 


\section{Results}

We perform our tests for brown dwarfs of spectral class L and T. Figure 1 shows the dust content (dust-to-gas ratio $\rho_{\mathrm{d}} / \rho_{\mathrm{g}}$ ) of the clouds calculated by different model approaches (Sect. 2). We observe similarities amongst the different cloud model results like the location of the inner cloud edge and the approximate location of the maximum dust content inside the cloud. However, differences are apparent like e.g. the pressure range covered by the clouds. We find the same behaviour for other opacity relevant cloud quantities like grain size and cloud material composition. It is therefore not surprising that the resulting spectra (Fig. 2) show a very similar general behaviour, like the absolute flux level, but vary in details. Figure 2 also demonstrates that the model atmosphere simulations for $\mathrm{T}_{\mathrm{eff}}=1800 \mathrm{~K}, \log g=5.0,[\mathrm{M} / \mathrm{H}]=0.0$ fall into two groups: those producing a thin cloud layer (Tsuji with $\mathrm{T}_{\text {crit }}=1900 \mathrm{~K}$, Homeier \& Allard) and those producing a thick cloud layer (Tsuji with $\mathrm{T}_{\text {crit }}=1700 \mathrm{~K}$, Marley \& Ackerman, Dehn/Helling\&Woitke (Dehn 2007)). It is interesting to realise that two entirely different mechanisms are responsible to move from one group to the other: Tsuji varies the geometrical cloud thickness by using a critical temperature $\mathrm{T}_{\text {crit }}$ for a constant grain size which results in an optically thinner/thicker cloud. The cloud models employed by Homeier \& Allard, Marley \& Ackerman, Dehn/Helling\&Woitke produce different amounts of dust (Fig. 1) and different grain size distributions across the cloud height causing an optically thiner/thicker cloud (not shown).

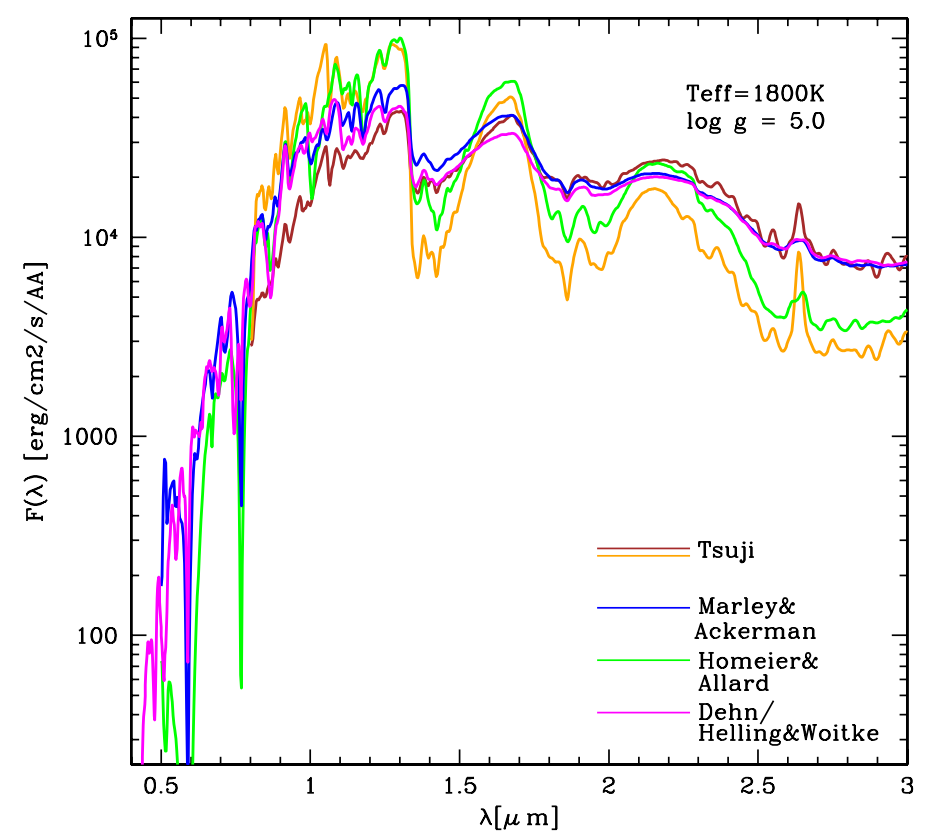

Figure 2. Results for Test case 2 (L dwarf): Spectral energy distribution between $0.5-3.0 \mu \mathrm{m}$ from different atmosphere codes utilising different cloud-model approaches for $\left(\mathrm{T}_{\mathrm{eff}}=1800 \mathrm{~K}\right.$, $\log g=5.0,[\mathrm{M} / \mathrm{H}]=0.0)$. Note, that the two Tsuji-cases demonstrate the influence of the cloud thickness: $\mathrm{T}_{\text {crit }}=1700 \mathrm{~K}$ (brown) - thick cloud, $\mathrm{T}_{\text {crit }}=1900 \mathrm{~K}$ (orange) - thin cloud. 


\section{Conclusion}

Our test case studies show that the results of our individual cloud models are comparable regarding general feature like the location of cloud base and the maximum dust-to-gas ratio. However, the cloud-model results differ if studied in more detail. It is therefore no surprise that the spectral energy distributions produced from our different $1 \mathrm{D}$ atmosphere simulations for a given parameter combination $\left(\mathrm{T}_{\text {eff }}, \operatorname{logg},[\mathrm{M} / \mathrm{H}]\right)$ differ in almost the entire wavelength range. It remains to quantify these differences in order to provide a general range of applicability for e.g. stellar parameter determinations inside and beyond the substellar regime.

\section{Acknowledgement:}

We thank all the participants of the workshop From Brown Dwarfs to Planets: Chemistry and Cloud formation which was supported by the Lorentz Center of the University Leiden, Nederlandse Organisatie voor Wetenschappelijk Onderzoek, The Netherlands research School for Astronomy, the Scottish University Physics Alliance, and European Space Agency. ChH acknowledges an IAU travel grant.

\section{References}

Ackerman, A. \& Marly, M. 2001, ApJ 556, 872

Allard, F., Allard, N. F., Homeier, D. et al. 2007, A\&A 474, L21

Allard, F., Guillot, T., Ludwig, H.-G., Hauschildt, P. H. et al. 2003, IAU 211, 325

Dehn, M. 2007, PhD Thesis, University Hamburg

Helling, Ch., Woitke, P., Thi, W.-F. 2008, submitted

Helling, Ch., Woitke, P. 2006, A\&A 455, 325

Ludwig, H.-G., Allard, F., Hauschildt, P.H. 2002, A\&A 395, 99

Sudarsky D., Burrows A., Hubeny I. 2003, ApJ 588, 1121

Tsuji, T. 2005, ApJ 621, 1033

Woitke P., Helling Ch. 2004, A\&A 414, 335

Woitke P., Helling Ch. 2003, A\&A 388, 297 